\title{
Effect of partitioning on the convergence properties of the Rayleigh-Schrödinger perturbation series
}

Zsuzsanna É. Mihálka, Ágnes Szabados, and Péter R. Surján

Citation: The Journal of Chemical Physics 146, 124121 (2017); doi: 10.1063/1.4978898

View online: http://dx.doi.org/10.1063/1.4978898

View Table of Contents: http://aip.scitation.org/toc/jcp/146/12

Published by the American Institute of Physics

\section{Articles you may be interested in}

Perspective: Found in translation: Quantum chemical tools for grasping non-covalent interactions

The Journal of Chemical Physics 146, 120901 (2017); 10.1063/1.4978951

Perspective: Computing (ro-)vibrational spectra of molecules with more than four atoms

The Journal of Chemical Physics 146, 120902 (2017); 10.1063/1.4979117

Incremental full configuration interaction

The Journal of Chemical Physics 146, 104102 (2017); 10.1063/1.4977727

A new efficient method for the calculation of interior eigenpairs and its application to vibrational structure problems

The Journal of Chemical Physics 146, 124101 (2017); 10.1063/1.4978581

Driven similarity renormalization group: Third-order multireference perturbation theory

The Journal of Chemical Physics 146, 124132 (2017); 10.1063/1.4979016

Development of an excited-state calculation method for large systems using dynamical polarizability: A divide-and-conquer approach at the time-dependent density functional level

The Journal of Chemical Physics 146, 124123 (2017); 10.1063/1.4978952

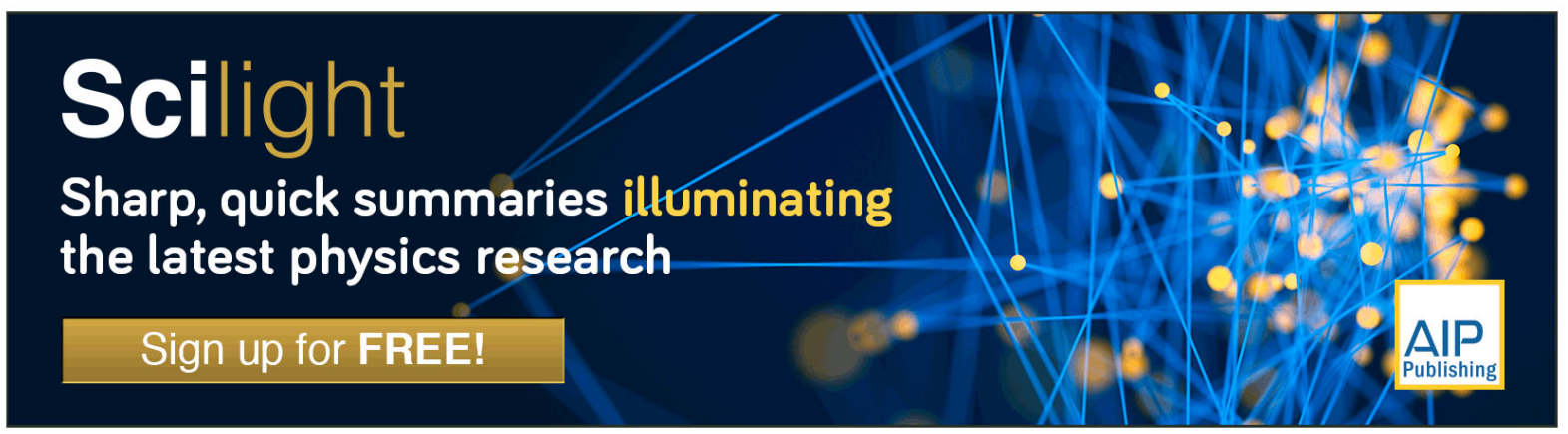




\title{
Effect of partitioning on the convergence properties of the Rayleigh-Schrödinger perturbation series
}

\author{
Zsuzsanna É. Mihálka, Ágnes Szabados, and Péter R. Surjána) \\ Laboratory of Theoretical Chemistry, Faculty of Science, Institute of Chemistry, Eötvös University, \\ H-1518 Budapest 112, P.O. Box. 32, Hungary
}

(Received 20 January 2017; accepted 7 March 2017; published online 28 March 2017)

\begin{abstract}
Convergence features of the Rayleigh-Schrödinger perturbation theory (PT) strongly depend on the partitioning applied. We investigate the large order behavior of the Møller-Plesset and Epstein Nesbet partitionings in comparison with a less known partitioning obtained by level shift parameters minimizing the norm of operator $\hat{Q} \hat{W}$, with $\hat{W}$ being the perturbation operator while $Q$ standing for the reduced resolvent of the zero order Hamiltonian $\hat{H}^{0}$. Numerical results, presented for molecular systems for the first time, indicate that it is possible to find level shift parameters in this way which convert divergent perturbation expansions to convergent ones in some cases. Besides numerical calculations of high-order PT terms, convergence radii of the corresponding perturbation expansions are also estimated using quadratic Padé approximants. Published by AIP Publishing. [http://dx.doi.org/10.1063/1.4978898]
\end{abstract}

\section{INTRODUCTION}

Perturbation theory (PT), besides its conceptual importance, represents a simple and efficient tool to approximate eigenvalues and eigenvectors of linear operators $\hat{H}$ systematically. ${ }^{1}$ The approximation goes as a power series of the perturbation parameter $\lambda$ defined by

$$
\hat{H}=\hat{H}^{0}+\lambda \hat{W}
$$

where $\hat{H}^{0}$ is the zero order operator while $\hat{W}$ is the perturbation.

Many quantum mechanical theories apply PT to deal with the energy operator (Hamiltonian). In quantum chemistry, an important field of application is the approximation of the correlation energy, for which the Rayleigh-Schrödinger (RS) PT is most often used, as it can be extensive. ${ }^{2,3}$ A huge number of examples are available showing the practical benefits of RSPT in this field, even at the few lowest orders of $\lambda$.

There is a disturbing problem, however. Given $\hat{H}$ and $\hat{H}^{0}$, one cannot predict whether or not the PT power series is convergent, and in fact, it was shown numerically to be divergent in a vast number of chemical applications (see, e.g., Refs. 4-7). Even if low order corrections serve as acceptable estimates, from a strict theoretical point of view it is not too elegant to use partial sums of an intrinsically divergent series.

Although the strict mathematical necessary and sufficient conditions of the convergence of the RSPT series are unknown, qualitative considerations may suggest some ideas. The solution of the RSPT problem ${ }^{8}$ at order $n$ can be written as

$$
\begin{aligned}
E^{(n)} & =\left\langle\Psi^{(0)}|\hat{W}| \Psi^{(n-1)}\right\rangle, \\
\left|\Psi^{(n)}\right\rangle & =\left[(\hat{Q} \hat{W})^{n}\right]_{c}\left|\Psi^{(0)}\right\rangle,
\end{aligned}
$$

\footnotetext{
a)Electronic address: surjan@chem.elte.hu
}

where $\Psi^{(n)}$ indicates the $n$th order eigenvector, and the reduced resolvent of $\hat{H}^{(0)}$ is

$$
\hat{Q}=\hat{P}\left(\hat{H}^{(0)}-E^{(0)}\right)^{-1} \hat{P}
$$

with $\hat{P}=1-\left|\Psi^{(0)}\right\rangle\left\langle\Psi^{(0)}\right|$ and with the subscript "c" in Eq. (3) indicating the connected terms of the operator product. Eq. (4) emphasizes the crucial role of operator product $(\hat{Q} \hat{W})$ in affecting the convergence features.

According to Eq. (4), the reduced resolvent is determined by the choice for the zero order Hamiltonian $\hat{H}^{0}$, i.e., by the partitioning. In electron correlation theory, one most often uses the so called Møller-Plesset (MP) partitioning, where $\hat{H}^{0}$ is chosen as the Fockian. One sometimes uses the Epstein-Nesbet (EN) partitioning, where $\hat{H}^{0}$ is constituted by the diagonal elements of the Hamiltonian matrix in the given basis set.

It is also known that level shift parameters (that change the partitioning) can influence convergence properties, too. Given all zero-order wave functions $\left|\Psi_{k}^{(0)}\right\rangle$, one can always redefine the partitioning (setting $\lambda=1$ ) like

$$
\hat{H}=\underbrace{\hat{H}^{0}+\sum_{k} \eta_{k}\left|\Psi_{k}^{(0)}\right\rangle\left\langle\Psi_{k}^{(0)}\right|}_{\hat{H}^{0^{\prime}}}+\underbrace{\hat{W}-\sum_{k} \eta_{k}\left|\Psi_{k}^{(0)}\right\rangle\left\langle\Psi_{k}^{(0)}\right|}_{\hat{W}^{\prime}} .
$$

The optimal choice of the level shift parameters $\eta_{k}$ has been discussed by several authors. ${ }^{7,9-30}$ In a previous paper, we proposed to minimize the square norm of operator $(\hat{Q} \hat{W})$ with respect to the level shift parameters in the hope of improving convergence features thereby. ${ }^{31}$ It was indeed shown that this technique is capable of converting divergent PT series of the quartic anharmonic oscillator to a convergent one (the series has zero radius of convergence if the harmonic oscillator Hamiltonian is used as the zero order).

In the present paper, we investigate the large order behavior of this latter partitioning for molecular systems when PT 
is used to estimate electron correlation starting from the single reference (Hartree-Fock) Hamiltonian, in comparison to the properties of MP and EN partitionings. In what follows, we briefly summarize the working equations, then present numerical results.

\section{WORKING EQUATIONS}

The square norm of operator $A$ is defined here as

$$
\|A\|^{2}=\operatorname{Tr}\left(A A^{\dagger}\right)
$$

which in a basis set representation is the two-norm or Frobenius norm of the matrix theory

$$
\|A\|^{2}=\sum_{i k} A_{i k} A_{i k}^{*}=\sum_{i k}\left|A_{i k}\right|^{2} .
$$

Evaluating $\|Q W\|^{2}$ with this definition, we get

$$
\begin{aligned}
\|Q W\|^{2} & =\sum_{i k}|\langle i|Q W| k\rangle|^{2}=\sum_{i k}\langle i|Q W| k\rangle\langle k|W Q| i\rangle \\
& =\sum_{i}\left\langle i\left|Q W^{2} Q\right| i\right\rangle=\sum_{i \neq 0} \frac{\left\langle i\left|W^{2}\right| i\right\rangle}{\left(E_{i}^{(0)}-E_{0}^{(0)}\right)^{2}} .
\end{aligned}
$$

With the level shifts (5), this transforms to

$$
\left\|Q^{\prime} W^{\prime}\right\|^{2}=\sum_{i \neq 0} \frac{\left\langle i\left|W^{2}\right| i\right\rangle-2 \eta_{i}\langle i|W| i\rangle+\eta_{i}^{2}}{\left(E_{i}^{(0)}-E_{0}^{(0)}+\eta_{i}\right)^{2}},
$$

where the level shift of the ground state, $\eta_{0}$, was set zero to fix the energy origin.

To minimize the norm with respect to $\eta_{k}$-s, we write

$$
\frac{\partial}{\partial \eta_{k}}\left\|Q^{\prime} W^{\prime}\right\|^{2}=0
$$

having the solution

$$
\eta_{k}=\frac{\left\langle k\left|W^{2}\right| k\right\rangle+\langle k|W| k\rangle\left(E_{k}^{(0)}-E_{0}^{(0)}\right)}{\langle k|W| k\rangle+\left(E_{k}^{(0)}-E_{0}^{(0)}\right)} .
$$

Using level shift parameters obtained from this equation results a new partitioning, which will be denoted by "QW" partitioning.

\section{ESTIMATING CONVERGENCE RADII}

As mentioned in the Introduction, the sufficient and necessary convergence conditions in RSPT are unknown. Numerical studies, more sophisticated than just monitoring partial sums, have been used however.

One ingenious idea is to consider the Hamiltonian as an operator-valued function of the perturbation parameter $\lambda$. As $\hat{H}(\lambda)$ varies with the value of perturbation parameter, so do its eigenvalues $E(\lambda)$. Allowing complex values for $\lambda$, properly denoted by letter $z$, the analyticity of the energy $E(z)$ as a complex function can be investigated numerically. 1,32 The idea is to find the singularity point of $E(z)$ closest to the origin, marking the convergence radius of PT. This is a rather complicated numerical procedure since it requires, at various values of $z$, to find the exact eigenvalue of the complex non-Hermitian Hamiltonian $\hat{H}(z)$. Therefore, as suggested by Goodson, ${ }^{33,34}$ one prefers to use Padé approximants ${ }^{35}$ to approximate $E(z)$.
The most frequently used Padé approximants are linear ones, approximating the sum of the PT series as the ratio of two polynomials. Accordingly, the only singularity they can exhibit is a simple pole. In many cases, however, nonanalyticity of $E(z)$ appears as a branching point on the complex plane. Goodson therefore suggested ${ }^{36}$ to apply quadratic Padé approximants $[\mathrm{K}, \mathrm{L}, \mathrm{M}]$ requiring

$$
P_{K}(z)+Q_{L}(z) S(z)+R_{M}(z) S^{2}(z) \approx 0
$$

to be satisfied, where $S(z)$ is the partial sum of the PT series, $P$, $Q$, and $R$ are $K, L$, and $M$-order polynomials whose coefficients are determined by requiring (11) to be satisfied for all values of $\mathrm{z}$.

The formal solution of Eq. (11) for $S(z)$ is

$$
S(z)=\frac{-Q_{L} \pm \sqrt{Q_{L}^{2}-4 P_{K} R_{M}}}{2 R_{M}}
$$

predicting a branch-point singularity if the discriminant is zero, that is, when

$$
Q_{L}^{2}-4 P_{K} R_{M}=0 .
$$

After solving the quadratic Padé problem Eq. (11), we get the coefficients of the discriminant which is a polynomial of degree at most $\max (2 L, K+M)$. We solve Eq. (13) numerically, find the roots of this polynomial, and choose the root $z \in \mathbb{C}$ which has the smallest modulus. This root, closest to the origin, will estimate the convergence radius. Clearly, since a Padé approximant is only an estimation of the converged value, the location of the branch-point is also only an approximation to the location of the true singularity. As shown by the numerical examples below, this estimation appears to be quite informative in the cases studied.

In this work, "diagonal" Padé approximants $[\mathrm{K}, \mathrm{K}, \mathrm{K}]$ will be used, i.e., we choose $K=L=M$.

\section{RESULTS}

This section reports high-order PT calculations on simple chemical systems for the correlation energy. The zero order Hamiltonian is originally chosen as the Fockian. The $n$th order results obtained from MP, and in some cases, the EN partitionings will be compared to those from the QW partitioning. The systems under study are the $\mathrm{H}_{2}$ molecule in a triple zeta polarized (TZP) basis at elongated interatomic distances 3.0 and $3.5 \AA$, the Ne atom in a relatively large $(13 \mathrm{~s} 7 \mathrm{p} 1 \mathrm{~d} / 7 \mathrm{~s} 4 \mathrm{p} 1 \mathrm{~d})$ basis including diffuse $\mathrm{d}$ and $\mathrm{s}$ functions, and the symmetrically stretched water molecule at various $\mathrm{OH}$ distances in a 6-31G* basis.

Since the numerical evaluation of Eq. (10) requires all diagonal matrix elements of operator $W^{2}$, it is most easily coded into a full CI program. We modified the program by Knowles and Handy ${ }^{37}$ to evaluate PT corrections in MP, EN, and QW partitionings.

\section{A. Stretched $\mathbf{H}_{2}$}

In Fig. 1, the first 40 order of total energies of $\mathrm{H}_{2}$ with bond distance $3.0 \AA$ are plotted in the MP and QW partitionings. Both PT results oscillate around the full CI line, but the MP values exhibit increasing oscillation amplitudes leading 


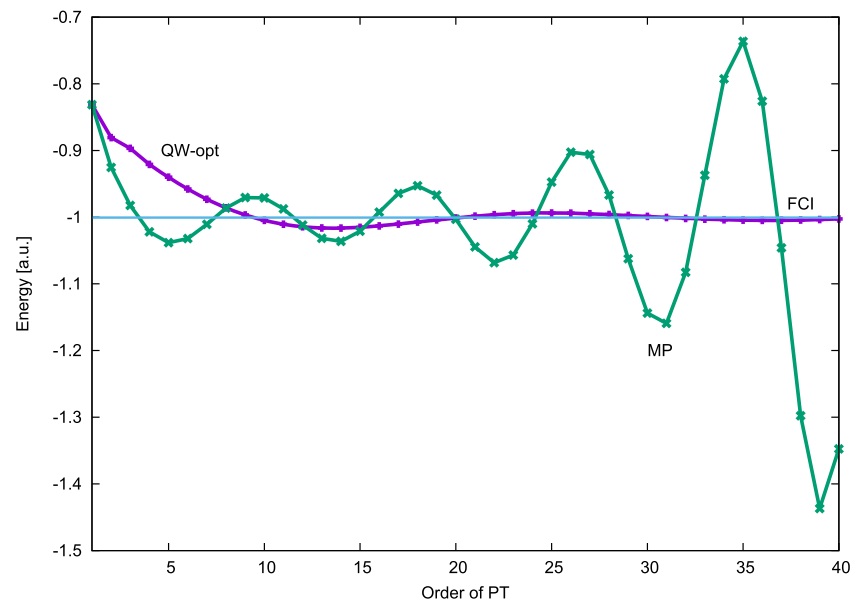

FIG. 1. Convergence of MP and QW perturbation energies for the $\mathrm{H}_{2}$ molecule at $3 \AA$ bond length.

to divergence, while the $\mathrm{QW}$ values show a damped behavior converging slowly to full CI. The price for this is that loworder results of QW are also significantly damped; thus the MP2 and MP3 energies induce the deceptive impression that the MP partitioning is superior.

In Fig. 2, we illustrate the convergence of the total energy of $\mathrm{H}_{2}$ at $3.5 \AA \mathrm{H}-\mathrm{H}$ distance in the QW partitioning. MPn results are not plotted here since they are pathologically divergent at such a large bond length. The primary figure shows the results up to order 400, while the inset is a zoom into the tail of the curve. The figure supports the impression that the QW PT series is, though slowly, convergent. This is an interesting feature of a single-reference method at such a large bond distance.

To analyze the conditions of convergence, we used quadratic diagonal $[6,6,6]$ Padé approximants estimating the location of eventual branch-points. Figure 3 shows that at $3.0 \AA$ bond length for the MPn series the singularity closest to the origin appears as a complex conjugate pair inside the unit circle, meaning that the convergence radius is less than 1 . On the other hand, the branch point for the QWn series is just outside the unit disk (the figure is somewhat misleading), appearing as a conjugate pair as well. This makes the estimate of the convergence radius just greater than 1 , in agreement with the former numerical assessment. The slow convergence is in accord with

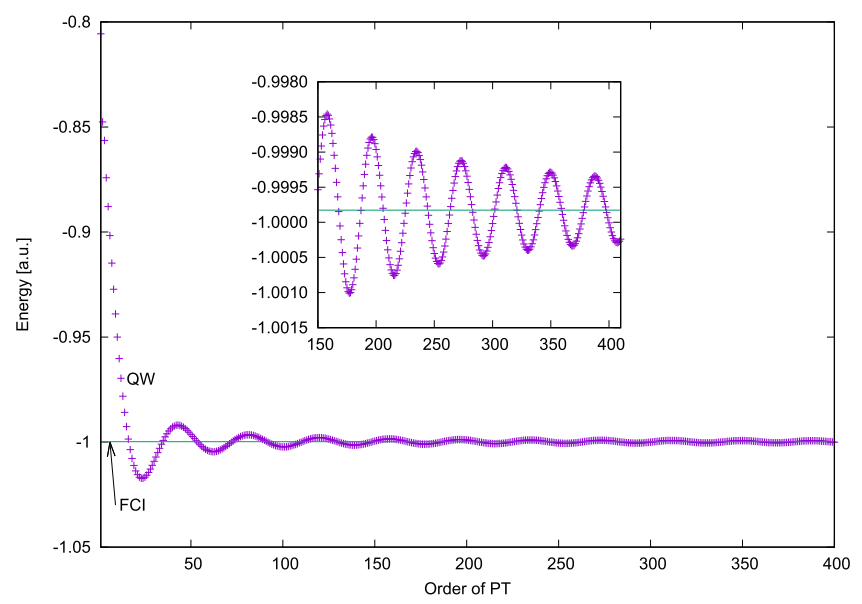

FIG. 2. The same as in Fig. 1, at $3.5 \AA$, for the QW partitioning. The inset is a zoom onto the orders $n>150$.

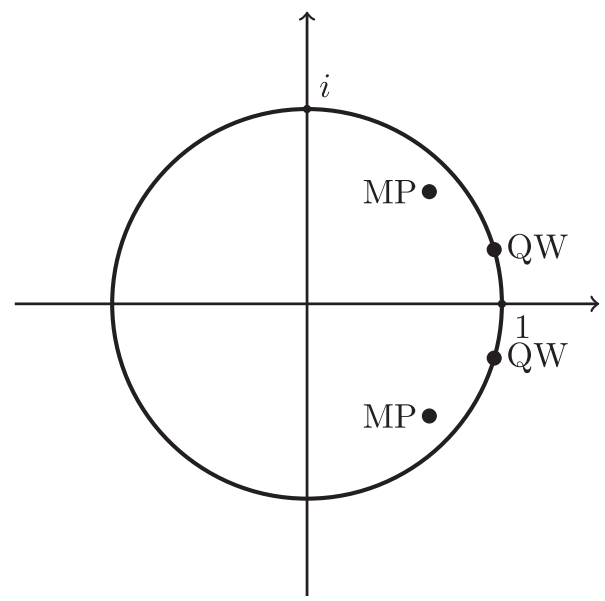

FIG. 3. Location of singularities (branch points) closest to the origin of the $E(z)$ function of $\mathrm{H}_{2}\left(\mathrm{R}_{\mathrm{HH}}=3.0 \AA\right)$ estimated by quadratic Padé approximants for the MP and QW partitionings.

the fact that a predicted singularity is very close to the unit circle.

\section{B. Ne atom}

Figure 4 compares MPn and QWn total energies in a $13 \mathrm{~s} 7 \mathrm{p} 1 \mathrm{~d}$ basis set contracted to $7 \mathrm{~s} 4 \mathrm{p} 1 \mathrm{~d}$. The picture is somewhat different from the case of $\mathrm{H}_{2}$ since here both the MP and QW results seem to be convergent up to order 8 . However, the convergence of MPn slows down above order 8, ending in a clear divergence above order 20 . In contrast, the QW results are nicely converging to the full $\mathrm{CI}$ value.

The radius of convergence of the MP and QW series was also estimated by the quadratic Padé approximation. We have used the diagonal $[4,4,4]$ approximation here; because some of the higher order contributions being very small, numerical instabilities for the $[6,6,6]$ Padé were encountered. Fig. 5 nicely shows that while the closest singularity for MP is inside the unit circle predicting divergence, the QW partitioning sufficiently enlarges the radius of convergence. In both cases, the singularities closest to the origin appear as complex conjugate pairs, with negative real parts ("backdoor intruders"2). The branch points for the MP partitioning lie almost on the real axis, and those for QW are also relatively close.

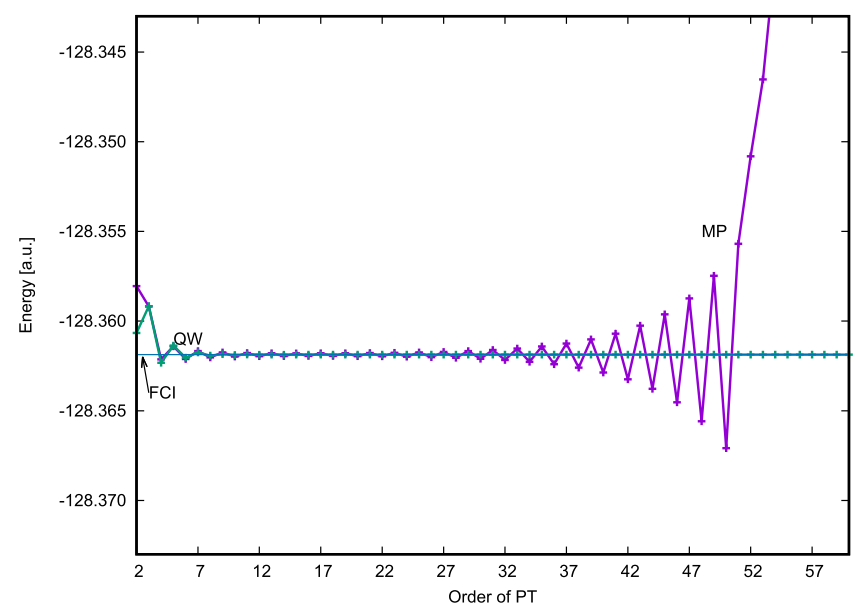

FIG. 4. Comparison of convergence of the MP and QW series for the Ne atom in a basis set containing diffuse functions. 


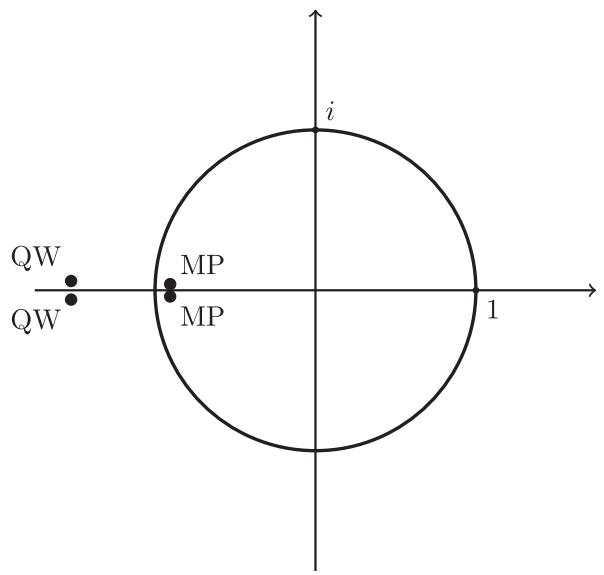

FIG. 5. Location of branch points closest to the origin, as predicted by $[4,4,4]$ Padé approximants, of the $E(z)$ function of the Ne atom (cf. Fig. 4).

\section{Water}

The MPn and QWn series were calculated in a 6-31G* basis set for water at several $\mathrm{O}-\mathrm{H}$ distances: equilibrium (eq) $\mathrm{O}-\mathrm{H}$ bond length and symmetrically stretched with $1.5 \mathrm{eq}$, 2.0 eq, 2.25 eq, and 2.5 eq, respectively. At shorter distances, the EN partitioning was also studied.

Figure 6 shows the energies obtained for all three partitionings at equilibrium geometry for water. In this case, each method is convergent. However, the QWn series converges slower than the MPn, as it does in most cases we encountered where both series were convergent. Even the EN partitioning gives a convergent result in this case, although it has an even slower convergence than QW results have (see inset). The MP results were omitted from this latter figure.

The EN, MP, and QW energies for 1.5 eq $\mathrm{O}-\mathrm{H}$ bond length can be seen in Figure 7. The MPn and QWn series are convergent but the EN result is divergent which can be seen better in the inset. The EN results are not convergent at any larger $\mathrm{O}-\mathrm{H}$ distances studied, for this reason these are not plotted.

At 2.0 eq O-H distance, we get a picture different from the former results (see Figure 8). Both the MPn and QWn results are convergent, but the series are not oscillating around the full

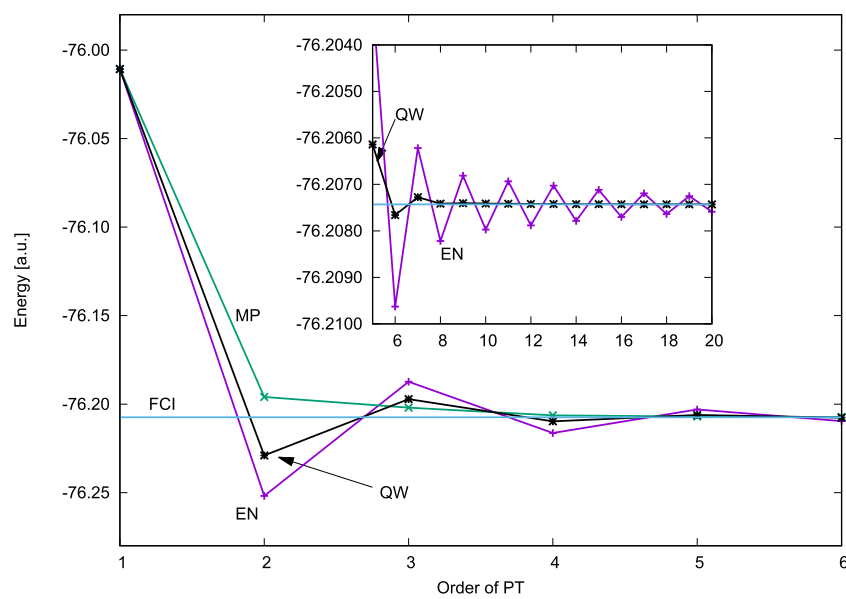

FIG. 6. Convergence of perturbation energies for the water molecule at equilibrium geometry in the MP, EN, and QW partitionings. The inset shows the behavior for orders $6-20$.

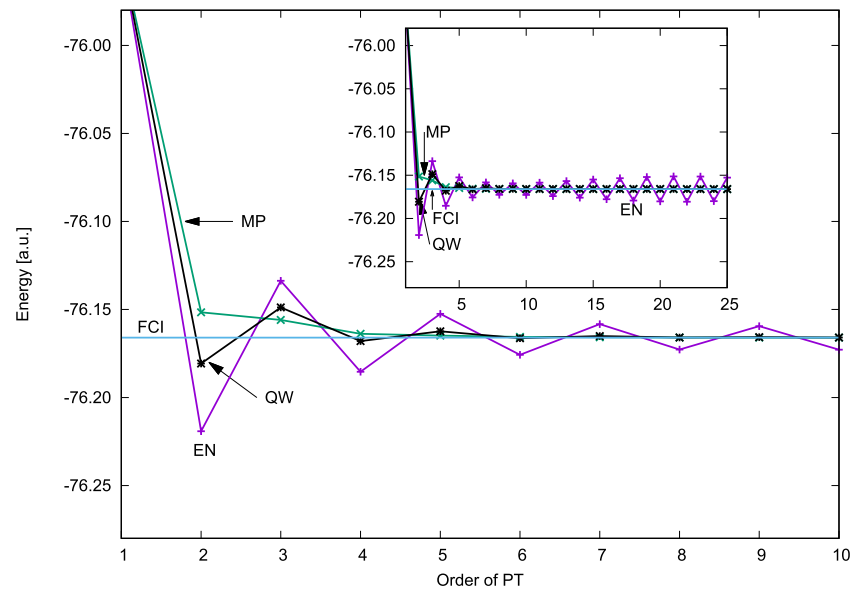

FIG. 7. EN, MP, and QW energies for symmetrically stretched water at 1.5 equilibrium bond length. Larger order contributions are shown by the inset.

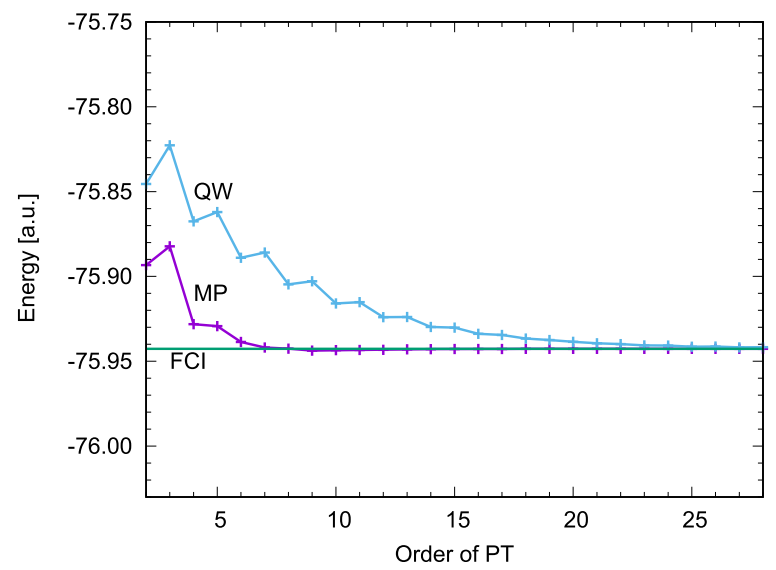

FIG. 8. The same as in Fig. 7, but for twice the equilibrium bond length.

configuration interaction (FCI) energy, rather they are monotonically decreasing. The slower convergence of the QWn results also manifests here.

Figure 9 compares MPn and QWn energies for the water molecule at a symmetrically stretched geometry with $2.25 \mathrm{eq}$ $\mathrm{O}-\mathrm{H}$ bond length. Even though at lower orders the MP series seems to give more accurate energies, going further in the summation it turns out that it is actually divergent. In comparison, the QWn series is convergent even at this prolongated bond

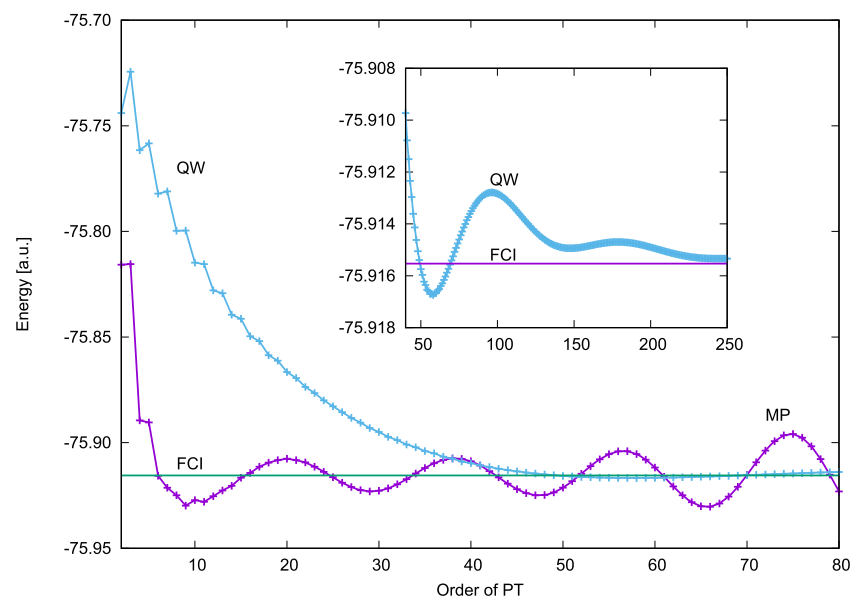

FIG. 9. The same as in Fig. 7, but for 2.25 times the equilibrium bond length. 

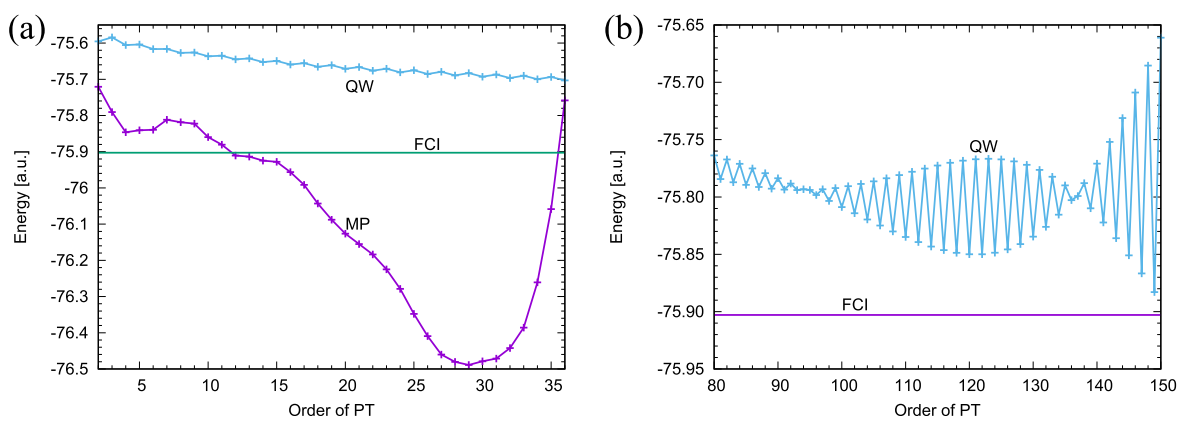

FIG. 10. The same as in Fig. 9, but for 2.5 times the equilibrium bond length; (a) convergence pattern at lower orders, (b) zoom into large-order behavior for QW. length, although the convergence is slow. The main figure is somewhat misleading in this case, since at this scale it seems like the QWn energy converged to the FCI energy at around the 50th order. This is not the case, as it can be seen in the inset. Still, the QWn results are convergent.

Finally, at 2.5 eq O-H distance, both methods fail to converge. Figure 10(a) shows the MPn and QWn series for this geometry. The divergence of the MPn results is obvious from this picture, and even though the QWn energies seem to slowly approach the exact result, going further the series deviates from full CI. Figure 10(b) shows the QWn results at higher orders. Instead of convergence, the QWn series exhibits an oscillating, divergent nature, resulting in a fractal-like picture. Note that the oscillation patterns are not around the exact (FCI) solution but around a strangely varying average value.

Convergence radii for these series were estimated using quadratic Padé approximants.

In Figure 11(a), we depict the branch points closest to the origin for EN partitioning at $1.0 \mathrm{eq}, 2.0 \mathrm{eq}$, and $2.5 \mathrm{eq} \mathrm{O}-\mathrm{H}$ bond lengths. To obtain these, in the first case $[5,5,5]$, while
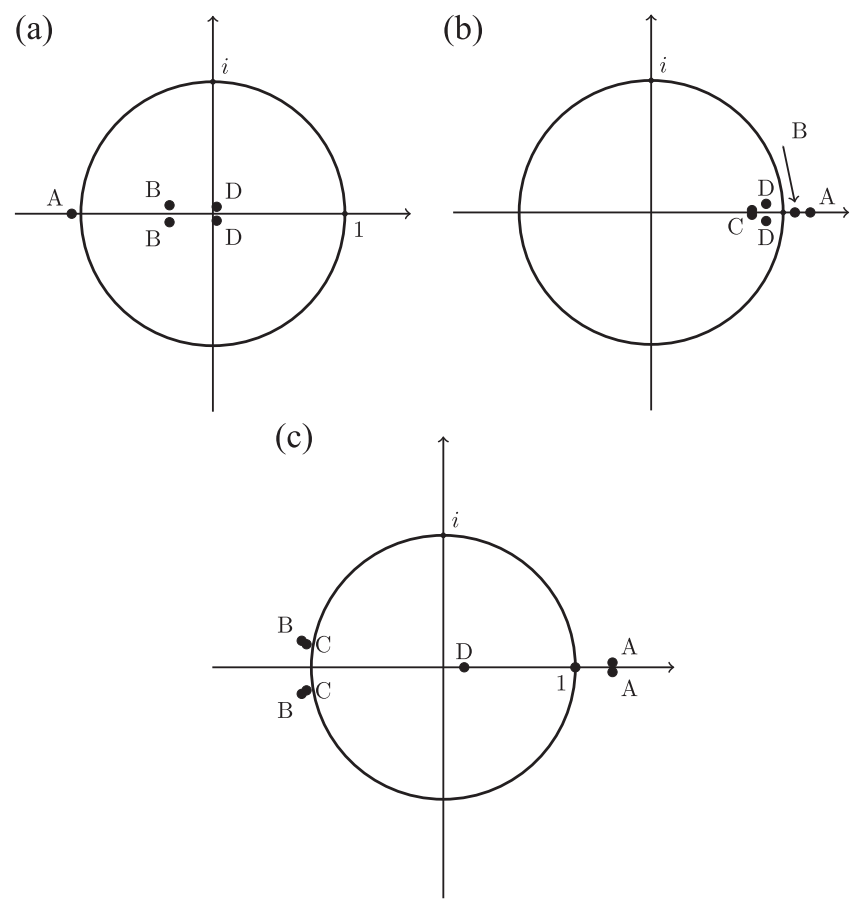

FIG. 11. Location of the closest branch points in different partitionings for symmetrically stretched water at different geometries (A: equilibrium, B: twice, C: 2.25 times, D: 2.5 times the equilibrium bond length): (a) EN partitioning, (b) MP partitioning, (c) QW partitioning. in the latter two cases, $[6,6,6]$ quadratic Padé approximants were used. The reason for the choice of a lower order approximant is the same as in the case of the Ne atom. At equilibrium $\mathrm{O}-\mathrm{H}$ distance, the branch point lies on the real axis, while at $2.0 \mathrm{eq}$ and $2.5 \mathrm{eq} \mathrm{O}-\mathrm{H}$ bond lengths the branch points are complex numbers. Their position is in agreement with the convergence results. At equilibrium geometry, the EN partitioning gave convergent results and the corresponding branch point is found outside of the unit circle. However, at greater distances the method fails to converge, and the branch points closest to the origin (i.e., the estimated convergence radii) lie within the unit circle.

Similarly, for the MPn series, [6,6,6] quadratic Padé approximants were used to determine the convergence radius at $2.0 \mathrm{eq}, 2.25 \mathrm{eq}$, and $2.5 \mathrm{eq} \mathrm{O}-\mathrm{H}$ distances, and the [5,5,5] Padé approximant was used to do the same at $1.0 \mathrm{eq} \mathrm{O}-\mathrm{H}$ bond length (Figure 11(b)). At shorter distances, the branch points lie outside the unit circle, and in these cases the MP results were convergent. At $2.25 \mathrm{eq}$ and $2.5 \mathrm{eq} \mathrm{O}-\mathrm{H}$ bond lengths, the estimated convergence radii are less than 1 . In two cases (denoted by "C" and "D"), the branch points are not on the real axis but show up as complex conjugate pairs.

The results for the QWn series are shown in Figure 11(c). Using $[6,6,6]$ quadratic Padé approximants, the branch points were estimated at the same geometries as for the MP case. For the $1.0 \mathrm{eq}, 2.0 \mathrm{eq}$, and $2.25 \mathrm{eq} \mathrm{O}-\mathrm{H}$ bond lengths, branch points are outside the unit circle, making the estimated convergence radii greater than 1 . At 2.5 eq bond length, the branch point " $D$ " lies inside the unit circle, resulting in the observed divergent behavior.

\section{Performance of linear and quadratic Padé approximants}

We select two illustrative cases, in which we show the performance of Padé approximants in MP and QW partitionings. One example is chosen among the nicely convergent cases (we selected the water molecule at equilibrium geometry). The other is the case when both MP and QW are divergent (water at $2.5 \mathrm{R}_{e q}$ ).

The relevant numbers are collected in Table I. Above each Padé approximant, we tabulate the PT $n$ value, $n$ being the highest order of PT used to construct the Padé approximant tabulated. That is, PT4 is compared to the [2,2] linear or the $[1,1,1]$ quadratic approximant. The items containing "n.r." indicate the cases which are not relevant the quadratic approximant predicting a complex energy at $\lambda=1$. 
TABLE I. Padé approximants for the water molecule in MP and QW partitionings, as compared to the partial sums (PTn) up to the order used to construct the approximant.

\begin{tabular}{|c|c|c|c|c|}
\hline \multirow{2}{*}{$\begin{array}{l}\text { Geometry } \\
\text { Partitioning }\end{array}$} & \multicolumn{2}{|c|}{$\mathrm{R}_{e q}$} & \multicolumn{2}{|c|}{$2.5 \mathrm{R}_{e q}$} \\
\hline & MP & QW & MP & QW \\
\hline PT4 & -76.206317 & -76.209708 & -75.846410 & -75.605454 \\
\hline Linear [2,2] Padé & -76.101234 & -76.207441 & -76.102737 & -75.605260 \\
\hline PT8 & -76.207417 & -76.207414 & -75.818721 & -75.627065 \\
\hline Linear $[4,4]$ Padé & -76.207404 & -76.207382 & -75.817055 & -75.7020016 \\
\hline PT12 & -76.207430 & -76.207421 & -75.910691 & -75.6449089 \\
\hline Linear $[6,6]$ Padé & -76.207430 & -76.207388 & -75.896083 & -75.7102526 \\
\hline PT4 & -76.206317 & -76.209708 & -75.846461 & -75.605454 \\
\hline Quadratic Padé [1,1,1] & -76.084627 & n.r. & -76.178874 & n.r. \\
\hline PT7 & -76.207370 & -76.207275 & -75.811638 & -75.616214 \\
\hline Quadratic Padé [2,2,2] & -76.207394 & -76.207306 & -75.823022 & n.r. \\
\hline PT10 & -76.207429 & -76.207412 & -75.859745 & -75.636470 \\
\hline Quadratic Padé [3,3,3] & -76.207430 & -76.207417 & -75.822073 & n.r. \\
\hline Full CI & \multicolumn{2}{|c|}{-76.207430} & \multicolumn{2}{|c|}{-75.902910} \\
\hline
\end{tabular}

The conclusion we can draw from the figures in Table I is that

(a) in the convergent case, both the linear and the quadratic approximants usually improve the PTn values, but the improvement is very small, and there are exceptions (like the [2,2] linear approximant);

(b) in the divergent case, the improvement shown by the Padé approximant is occasionally quite significant, but in other cases the extrapolated values are much worse than simple partial sums.

TABLE II. Role of branch point locations $r^{*}$ on the error of low-order PT results $\mathrm{E}(n)-\mathrm{E}(\mathrm{FCI})$ (atomic units) in $\mathrm{MP}$ and $\mathrm{QW}$ partitionings for the water molecule at various $C_{2 v}$ geometries.

\begin{tabular}{|c|c|c|c|c|c|}
\hline$R_{e q} \times$ & 1.0 & 1.5 & 2.0 & 2.25 & 2.5 \\
\hline \multicolumn{6}{|c|}{ MP partitioning } \\
\hline $\operatorname{Re}\left(r^{*}\right)$ & 1.2059 & 1.4063 & 1.0890 & 0.7637 & 0.8711 \\
\hline $\operatorname{Im}\left(r^{*}\right)$ & 0.0010 & 0.0000 & 0.0112 & 0.0177 & 0.0652 \\
\hline$r_{\text {conv }}$ & 1.2059 & 1.4063 & 1.0891 & 0.7639 & 0.8735 \\
\hline \multicolumn{6}{|c|}{$\mathrm{E}(n)-\mathrm{E}(\mathrm{FCI})$} \\
\hline MP2 & 0.011474 & 0.014429 & 0.049365 & 0.099774 & 0.182352 \\
\hline MP3 & 0.005465 & 0.010088 & 0.060451 & 0.100048 & 0.112276 \\
\hline MP4 & 0.001113 & 0.002174 & 0.014580 & 0.026051 & 0.056450 \\
\hline MP5 & 0.000424 & 0.001155 & 0.013403 & 0.025130 & 0.062209 \\
\hline MP6 & 0.000122 & 0.000420 & 0.004023 & -0.000144 & 0.063420 \\
\hline MP7 & 0.000060 & 0.000198 & 0.000735 & -0.005825 & 0.091272 \\
\hline \multicolumn{6}{|c|}{ QW partitioning } \\
\hline $\operatorname{Re}\left(r^{*}\right)$ & 1.28158 & 1.1764 & -1.0729 & -1.03642 & 0.1584 \\
\hline $\operatorname{Im}\left(r^{*}\right)$ & 0.03607 & 0.0000 & 0.2012 & 0.17467 & 0.0000 \\
\hline$r_{c o n v}$ & 1.28209 & 1.1764 & 1.0916 & 1.05103 & 0.1584 \\
\hline \multicolumn{6}{|c|}{$\mathrm{E}(n)-\mathrm{E}(\mathrm{FCI})$} \\
\hline QW2 & -0.021545 & -0.014683 & 0.097166 & 0.1715330 & 0.307652 \\
\hline QW3 & 0.010220 & 0.017169 & 0.119982 & 0.1911208 & 0.318528 \\
\hline QW4 & -0.002278 & -0.001974 & 0.075089 & 0.1539849 & 0.297456 \\
\hline QW5 & 0.001290 & 0.003604 & 0.080633 & 0.1571811 & 0.298915 \\
\hline
\end{tabular}

\section{E. Role of the location of branch points}

An interesting fact seen in Table II is that the deviation of $r_{\text {conv }}$ from 1 is not strictly correlated to the apparent speed of convergence. For example, comparing geometries 1.0 and $1.5 R_{e q}$ in the MP partitioning, the former geometry shows a better convergence (as of course, chemically expected), but the branch point is farther from 1 in the latter case. This can be true because we do not investigate the same analytic function $E(z)$, but we compare two different systems (water at different geometries).

Another observation is that in all cases tabulated the imaginary part of $r^{*}$ is rather small: the branch point closest to the origin lies close to the real axis. The small variations shown by $\operatorname{Im}\left(r^{*}\right)$ do not seem to have any influence onto the convergence patterns.

\section{CONCLUSION}

As seen from the example of the water molecule, the EN partitioning exhibits the least favorable convergence properties. In comparison with MP, this is clearly due to the fact that EN denominators (essentially Hartree-Fock excitation energies) are smaller quantities than MP denominators (orbital energy differences).

Using the Hartree-Fock determinant as the zero order reference state, we found that minimizing the norm $\| \mathrm{QW \|}$ in RSPT with respect to level shift parameters, it is possible to convert diverging PT series into converging ones. This happens even for molecular geometries far from equilibrium, i.e., in cases which are difficult for the single reference perturbation theory. Of the numerically investigated cases, it was only a single example $\left(C_{2 v}\right.$ water with $\left.R_{\mathrm{OH}}=2.5 \mathrm{eq}\right)$, when the PT in the optimized QW partitioning remained divergent.

In all cases studied, the numerical findings on the apparent convergence or divergence were in accord with the convergence radii estimated by quadratic Padé approximants.

The price for ensuring the convergence is that loworder QW results are in most cases inferior to MP or EN energies. This is natural since minimizing $\| \mathrm{QW \|}$ results 
enlarged energy denominators which damp the PT but make individual contributions smaller.

\section{ACKNOWLEDGMENTS}

This work was partly supported by the Grant No. NKFIH-K115744. Á.Sz. acknowledges support by the János Bolyai Research Scholarship of the Hungarian Academy of Sciences.

${ }^{1}$ T. Kato, Perturbation Theory for Linear Operators (Springer, Berlin, 1966).

${ }^{2}$ T. Helgaker, P. Jørgensen, and J. Olsen, Molecular Electronic-Structure Theory (John Wiley \& Sons Ltd., England, 2000).

${ }^{3}$ I. Mayer, Simple Theorems, Proofs, and Derivations in Quantum Chemistry (Kluwer, New York, 2003).

${ }^{4}$ J. Olsen, B. O. Roos, P. Jørgensen, and J. Aa. Jensen, J. Chem. Phys. 89, 2185 (1988).

${ }^{5}$ M. L. Leininger, W. D. Allen, H. F. Schaefer, and C. David Sherrill, J. Chem. Phys. 112, 9213-9222 (2000).

${ }^{6}$ A. V. Sergeev, D. Z. Goodson, S. E. Wheeler, and W. D. Allen, J. Chem. Phys. 123, 064105 (2005).

${ }^{7}$ B. Forsberg, Z. He, Y. He, and D. Cremer, Int. J. Quantum Chem. 76, 306-330 (2000)

${ }^{8}$ J. Paldus and J. Č́žzek, Adv. Quantum Chem. 9, 105-197 (1975).

${ }^{9}$ S. Zarrabian and J. Paldus, Int. J. Quantum Chem. 38, 761 (1990).

${ }^{10} \mathrm{~S}$. Wilson, K. Jankowski, and J. Paldus, Int. J. Quantum Chem. 23, 1781-1802 (1983).

${ }^{11}$ D. Hegarty and M. A. Robb, Mol. Phys. 37, 1455 (1979).

${ }^{12}$ U. Kaldor, Int. J. Quantum Chem. 28, 103 (1985).

${ }^{13}$ I. Shavitt and L. T. Redmon, J. Chem. Phys. 73, 5711 (1980).

${ }^{14}$ J. Mášik, I. Hubač, and P. Mach, Int. J. Quantum Chem. 53, 207 (1995).

${ }^{15}$ V. I. Alexandrov, A. V. Zaitevskii, and A. I. Dementev, Chem. Phys. Lett. 218, 206 (1993).
${ }^{16}$ E. Feenberg, Phys. Rev. 103, 1116 (1956).

${ }^{17}$ P. Goldhammer and E. Feenberg, Phys. Rev. 101, 1233 (1955).

${ }^{18}$ A. T. Amos, J. Chem. Phys. 52, 603 (1970).

${ }^{19}$ K. Dietz, C. Schmidt, M. Warken, and B. A. Hess, J. Phys. B: At., Mol. Opt. Phys. 26, 1885 (1993).

${ }^{20}$ K. Dietz, C. Schmidt, M. Warken, and B. A. Hess, J. Phys. B: At., Mol. Opt. Phys. 26, 1897 (1993).

${ }^{21}$ J. P. Finley, R. K. Chaudhuri, and K. F. Freed, J. Chem. Phys. 103, 4990 (1995).

${ }^{22}$ J. P. Finley, R. K. Chaudhuri, and K. F. Freed, Phys. Rev. A 54, 343 (1996).

${ }^{23}$ R. K. Chaudhuri, J. P. Finley, and K. F. Freed, J. Chem. Phys. 106, 4067 (2001).

${ }^{24}$ Á. Szabados and P. R. Surján, Chem. Phys. Lett. 308, 303 (1999).

${ }^{25}$ P. R. Surján and Á. Szabados, J. Chem. Phys. 112, 4438 (2000).

${ }^{26}$ H. A. Witek, H. Nakano, and K. Hirao, J. Chem. Phys. 118, 8197-8206 (2003).

${ }^{27}$ H. A. Witek, H. Nakano, and K. Hirao, J. Comput. Chem. 24, 1390-1400 (2003).

${ }^{28}$ Z. Rolik, Á. Szabados, and P. R. Surján, J. Chem. Phys. 119, 1922 (2003).

${ }^{29}$ P. R. Surján, Á. Szabados, and Zs. Szekeres, Int. J. Quantum Chem. 90, 1309-1320 (2002).

${ }^{30}$ P. R. Surján and Á. Szabados, “Appendix to 'studies in perturbation theory': The problem of partitioning," in Fundamental World of Quantum Chemistry, A Tribute to the Memory of Per-Olov Löwdin, Vol. 3, edited by E. J. Brändas and E. S. Kryachko (Kluwer, Dordrecht, 2004), pp. 129-185.

${ }^{31}$ P. R. Surján and Á. Szabados, Collect. Czech. Chem. Commun. 69, 105 (2004).

${ }^{32}$ W. H. Adams, Int. J. Quantum Chem. 109, 3844-3857 (2009).

${ }^{33}$ D. Z. Goodson, J. Chem. Phys. 112, 4901 (2000).

${ }^{34}$ A. V. Sergeev and D. Z. Goodson, J. Phys. A: Math. Gen. 31, 4301 (1998).

${ }^{35}$ H. Padé, J. Math. Pures Appl. 10, 291-329 (1894).

${ }^{36}$ D. Z. Goodson, "Resummation methods," Wiley Interdiscip. Rev.: Comput. Mol. Sci. 2, 743-761 (2012).

${ }^{37}$ P. J. Knowles and N. C. Handy, Chem. Phys. Lett. 111, 315 (1984). 